\title{
Pure Tibiotalar Dislocation with Talus Bone Contusion in a Volleyball Player: A case report
}

\author{
by N.E. Koukoulias, MD, $\mathrm{PhD}^{1 凶}$, S.G. Papastergiou, MD, $\mathrm{PhD}^{2}$, P. Panagopoulos, $\mathrm{MD}^{3}$, T. Dimitriadis, $\mathrm{MD}^{4}$, \\ P. Koumis, $\mathrm{MD}^{5}$
}

The Foot and Ankle Online Journal 2 (9): 2

We present a case of closed, pure tibiotalar dislocation. A 21 year-old volleyball player sustained a pure ankle dislocation after jumping up at the net and landing on the floor with a fully plantarflexed foot that was inverted during landing. After closed reduction, a short leg cast was applied for ankle immobilization. Magnetic resonance imaging demonstrated rupture of the anterolateral capsuloligamentous structures and talus bone contusion. The cast was removed at two months post-injury and full weight-bearing was permitted at three months. The patient returned to pre-injury level of activity at 6 months. Three years post-injury, the patient is asymptomatic, without signs of instability or degenerative arthritis.

Key words: Tibiotalar dislocation, talus contusion, magnetic resonance imaging

Accepted: August, 2009

Published: September, 2009

This is an Open Access article distributed under the terms of the Creative Commons Attribution License. It permits unrestricted use, distribution, and reproduction in any medium, provided the original work is properly cited. @The Foot and Ankle Online Journal (www.faoj.org)

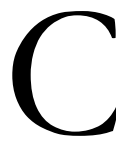

losed tibiotalar dislocation without concomitant malleolar fracture or syndesmotic injury is a rare. ${ }^{1-5}$ Ankle sprain is often accompanied by chondral or osteochondral injuries that may result in persisted pain and disability. ${ }^{6,7}$

This is the first case of pure ankle dislocation in the literature, with documented talus bone contusion. This finding is crucial for proper treatment planning in order to avoid complications like tibiotalar degenerative arthritis and talar avascular necrosis.

\section{Case Report}

A 21 year-old volleyball player injured his left ankle during a second league game. The injury occurred after jumping up at the net and landing on the floor with a fully plantarflexed foot that was inverted during landing. (Fig.1)

Address correspondence to: N.E. Koukoulias, 161 Ethnikis Antistasis St, 55134, Thessaloniki, Greece, Tel: 00302310493552.

\footnotetext{
1,2,3,4,5 Department of Orthopaedics, Sports Injuries Unit, “Agios Pavlos” General Hospital, Thessaloniki, Greece.
}

Physical examination in the emergency room showed a deformed ankle without neurovascular deficit of the foot. Radiographs of the ankle demonstrated ankle dislocation without concomitant syndesmotic injury or malleolar fracture.

The ankle was reduced under general anaesthesia. After close reduction, a short leg cast was used for ankle immobilization. (Fig. 2) The patient was then further investigated with magnetic resonance imaging (MRI) to fully explore the structures that were injured. MRI demonstrated rupture of the anterior capsule and anterior talo-fibular ligament and talus bone contusion. (Fig. 3) The cast was kept on for two months. After the cast removal, the patient was referred to the physiotherapist and range of motion exercises were initiated along with partial weightbearing. Three months post-injury, full weight-bearing was permitted.

The patient returned to the pre-injury level of activity at six months post-injury. At physical examination there were no signs of residual ligamentous laxity and no ankle effusion and tenderness. 


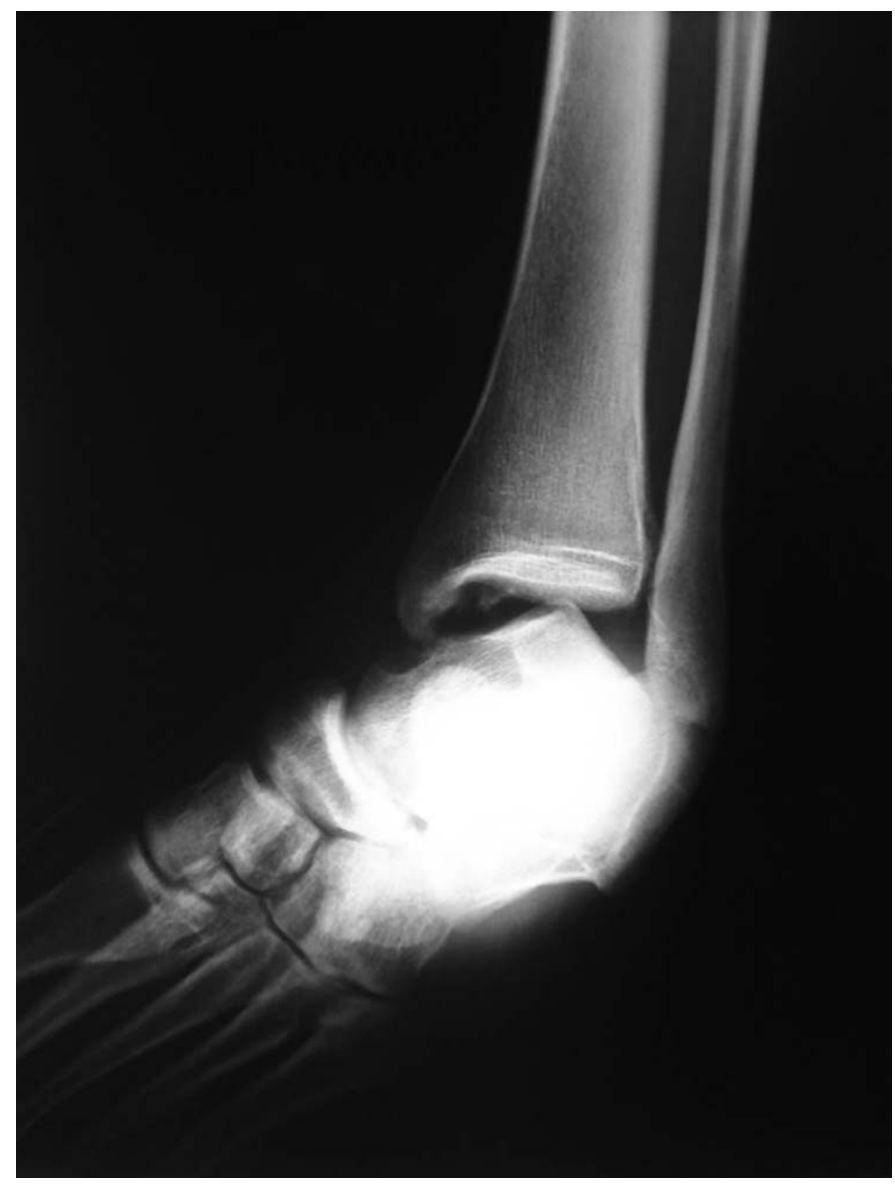

Figure 1 The anteroposterior view demonstrates tibiotalar joint dislocation without concomitant malleolar fracture or syndesmotic injury.

Three years post-injury the patient is still asymptomatic without signs of tibiotalar degenerative arthritis or talar avascular necrosis in ankle radiographs.

\section{Discussion}

In 1939, Wilson, et al., were the first to report on ankle dislocation without fracture. Since this time, closed pure ankle dislocations were sporadically reported, while other authors reported open dislocations or dislocations accompanied by tibiofibular diastasis (syndesmotic injury) or mixed data. ${ }^{1-5}$

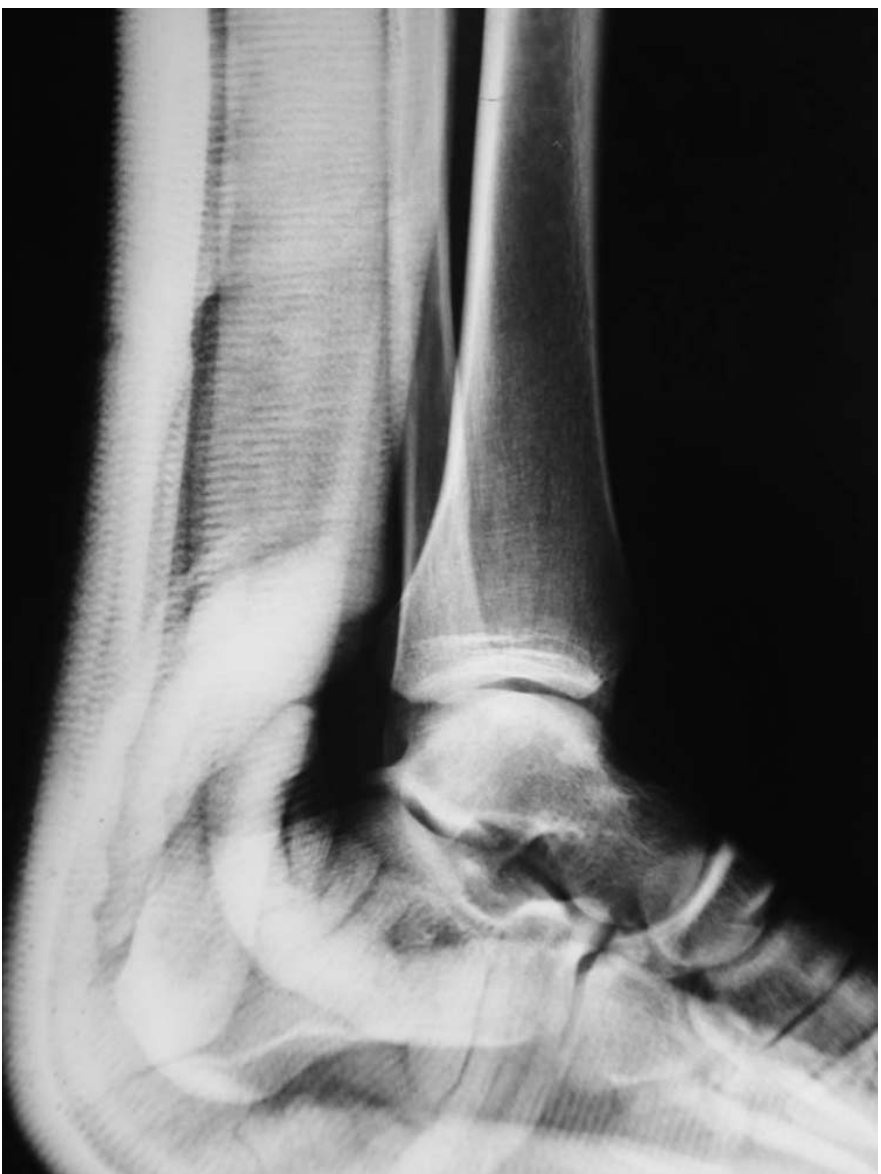

Figure 2 Lateral view of the tibiotalar joint after closed reduction.

Interestingly, none of these cases was investigated with MRI. All patients had conventional radiography that, generally, cannot reveal chondral or osteochondral injuries. ${ }^{6}$ In comparison, MRI is a high sensitive modality for detecting this kind of pathology. ${ }^{6} \quad$ Sijbrandij, et al., found chondral and osteochondral injuries in 18\% of their patients with ankle sprain. ${ }^{6} \quad$ Ly and Fallat reported that these injuries are responsible for persisted pain and disability after ankle sprain. ${ }^{7}$ It is assumed that incidence and degree of bone contusion depends on the severity of the sprain and the residual instability that could cause recurrent sprains. ${ }^{6}$ The natural history of ankle bone contusion is still unknown. The evolution towards tibiotalar degenerative arthritis or talar avascular necrosis has not been extensively studied in the literature. 


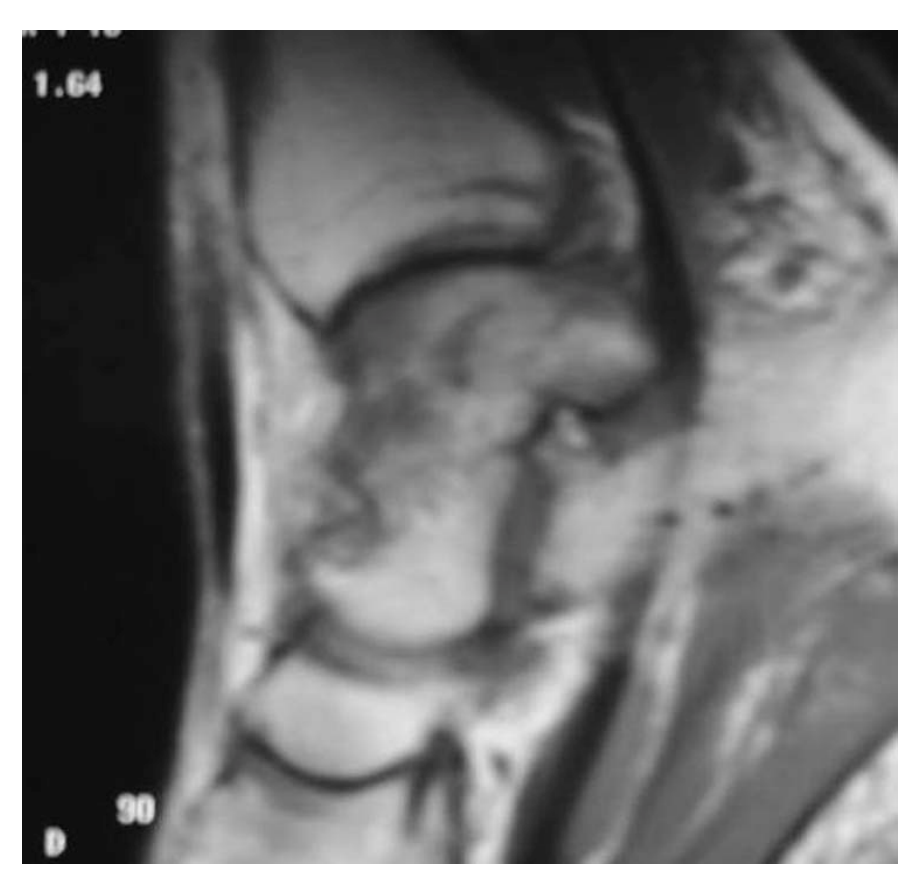

Figure 3 T1 sagittal image demonstrates talus bone contusion.

Sijbrandij, et al., found that bone contusion was resolved in $1-4$ months after injury, but in some cases persisted for more than 4 months. ${ }^{6}$ In contrast, degenerative arthritis after ankle dislocation should be anticipated in $25 \%$ of cases. ${ }^{5}$ As a result, MRI is mandatory for accurate diagnosis and proper treatment planning in these severe injuries.

Most of the authors reported on ankle dislocation without fracture, recommend conservative treatment using a short leg cast for $4-6$ weeks and full weight bearing at $3-6$ weeks. Nevertheless, only Tayamaz and Gunal reported long-term results of their case. ${ }^{4}$ Consequently, no safe conclusions can be drawn regarding the success of the treatment. In our opinion, these cases should be treated individually, based primarily on an accurate diagnosis provided by MRI. The length of immobilization should be $6-8$ weeks to allow soft tissue healing. Full weight-bearing should be decided according to the presence and degree of chondral and osteochondral injury in combination with joint effusion and tenderness assessed in physical examination. It is clear that longterm follow up studies are needed to establish treatment guidelines for these severe injuries.

\section{References}

1. Wilson MJ, Michele AA, Jacobson EW: Ankle dislocations without fracture. J Bone Joint Surg 21A: 198 - 204, 1939. 2. Uyar M, Tan A, Işler M, Cetinus E: Closed posteromedial dislocation of the tibiotalar joint without fracture in a basketball player. Br J Sports Med 38 (3):342 - 343, 2004.

3. Soyer AD, Nestor BJ, Friedman SJ: Closed posteromedial dislocation of the tibiotalar joint without fracture or diastasis: a case report. Foot Ankle Int 15 (11):622 - 624, 1994.

4. Taymaz A, Gunal I: Complete dislocation of the talus unaccompanied by fracture. J Foot Ankle Surg 44 (2):156 - 158, 2005.

5. Elisé S, Maynou C, Mestdagh H, Forgeois P, Labourdette P: Simple tibiotalar luxation. A proposal of 16 cases. Acta Orthop Belg 64 (1):25 - 34, 1998.

6. Sijbrandij ES, van Gils AP, Louwerens JW, de Lange EE:

Posttraumatic subchondral bone contusions and fractures of the talotibial joint: occurrence of "kissing" lesions. Am J Roentgenol 175 (6):1707 - 1710, 2000.

7. Ly PN, Fallat M: Transchondral contusions of the talus: a review of 64 surgical cases. J Foot Surg 32 (4):352 - 374, 1993. 